\title{
¿QUÉ TRANSPARENCIA REQUIERE EL GOBIERNO ABIERTO?
}

\author{
Freddy Mariñez Navarro \\ Instituto Tecnológico y de Estudios Superiores de Monterrey, México \\ fmarinez@itesm.mx
}

\section{RESUMEN}

Este artículo aborda la transparencia desde la perspectiva del Gobierno Abierto. Se analizan los nuevos enfoques de la transparencia producto de los procesos y reformas de la gestión pública así como de los avances de las Tecnologías de Información y Comunicación (TICs). De esta manera, el Gobierno Abierto como modelo de gestión y visto como plataforma de democracia colaborativa se convierte en el contexto fundamental de la transparencia. En dos puntos explicamos esta relación. Primeramente, nos aproximamos al concepto del Gobierno Abierto, haciendo un mapeo de las definiciones que han surgido últimamente y sus implicaciones tanto en la colaboración, participación y transparencia. Seguido, nos adentramos a la nueva propuesta de transparencia con la siguiente interrogante: ¿qué transparencia necesita el Gobierno Abierto?

Palabras clave: Transparencia, Gobierno Abierto, Política Pública, Transparencia focalizada. 


\title{
WHICH TRANSPARENCY DOES THE OPEN GOVERNMENT REQUIRE?
}

\begin{abstract}
This article discusses transparency from the Open Government perspective. New approaches of transparency as a result of processes and reforms of public management, as well as progress made in Information and Communications Technologies (ICTs), are analyzed. In this manner, Open Government, as a management model and seen as a platform for collaborative democracy, becomes the fundamental context for transparency. We explained this relationship in two points. Firstly, we approach the concept of Open Government, by mapping the definitions which have recently emerged, as well as its implications for collaboration, participation and transparency. Secondly, we advance a new proposal for transparency with the following question: which transparency does the Open Government need?
\end{abstract}

Keywords: Transparency, Open Government, Public Policy, Targeted transparency. 


\section{UNA APROXIMACIÓN AL CONCEPTO DE GOBIERNO ABIERTO}

El Gobierno Abierto nos brinda múltiples definiciones y en cada una se identifican tendencias con un abordaje teórico muy particular. Es así que algunos sostienen que un Gobierno Abierto es resultado del cambio tecnológico, donde la web 2.0 juega un rol fundamental y el centro de su análisis son las tecnologías de información. A su vez, otros enfatizan la importancia de concebir el Gobierno Abierto como un cambio en los valores del servicio público y en la organización, en síntesis, se traduce en una nueva cultura organizacional. También se explica que el Open Data, o mejor conocido como datos abiertos, viene a revolucionar el concepto de transparencia tal como se había venido implementando. Contar con información que puede ser transformada para un fin mayor al interés particular, resulta en una nueva relación entre productor de información y receptor de la misma.

Si se parte de la definición de Meijer, Curtin y Hillebrandt (2012), en la cual la apertura del gobierno es la medida por la que los ciudadanos pueden monitorear e influir los procesos del gobierno a través del acceso a la información gubernamental y el acceso a la arena de la hechura de las decisiones, entonces, a partir de esta apertura, podríamos decir que la producción de datos e información tiene valor en sí misma. Ello se explica por la razón que un dato por sí mismo no es nada, sino hasta cuando se juntan diversos datos que luego se convierten en información. Además, si a esta información somos capaces de darle interpretación, estaríamos entonces hablando de conocimiento. Quizás ése sea el espíritu de Open Data. "Los datos que en sí mismos no ofrecen ningún valor por lo que hay que dotarlos de significado para convertirlos en conocimiento válido y con valor para los demás. Visto así, es el objetivo esencial del Gobierno Abierto" (Linares 2010: 62).

Guardián Orta (2010) nos presenta una propuesta de Gobierno Abierto definida como:

[...] la doctrina política que sostiene que la actividad del gobierno y de la administración del Estado debe estar abierta a todos los niveles para el escrutinio eficaz del público y de su supervisión. En su más amplia concepción se opone a la razón del Estado de legitimar como secreto de Estado cierta información aduciendo temas de seguridad (2010: 76).

La definición de Calderón y Lorenzo (2010) también hace aportaciones al tema del Gobierno Abierto cuando expresan que éste es aquel: 
[...] que entabla una constante conversación con los ciudadanos con el fin de escuchar lo que ellos dicen y solicitan, que toma decisiones basadas en sus necesidades y preferencias, que facilita la colaboración de los ciudadanos y funcionarios en el desarrollo de los servicios que presta y que comunica todo lo que decide y hace de forma abierta y transparente (2010: 11).

Otra visión es la de Ramírez Alujas (2011), quien deja claro que el término Gobierno Abierto no es nuevo y se usó por primera vez en el espacio político británico. En su concepción original, se trataba de un gobierno que buscaba "abrir las ventanas" del sector público hacia el escrutinio ciudadano, con el objeto de combatir el secreto gubernamental y reducir la opacidad burocrática. Taewoo Nan (2012) ha llegado a la conclusión a través de estudios empíricos que:

[...] dos palabras de moda que actualmente están infiltrando el sector público son Gobierno Abierto (Open Government) y Government 2.0. En la trayectoria del desarrollo de e-gobierno, el Gobierno Abierto y el Gobierno 2.0 parecen ser, respectivamente, sus nuevos medios. Es una realidad no sólo en los Estados Unidos, sino también en otras democracias avanzadas, así como en ciertos países en vía de desarrollo. La plataforma y la norma para la tecnología permitida por el gobierno se están moviendo del e-gobierno (Government 1.0) al Gobierno Abierto (Government 2.0) (2012: 347).

En septiembre del año 2011, se constituyó el Open Government Partnership. Esta red reúne las diferentes iniciativas de una cantidad significativa de los gobiernos del mundo. Su misión es introducir a los gobiernos así como las organizaciones no gubernamentales y las empresas privadas con experiencia, habilidades y tecnologías a asistir a los países tanto conceptualmente como en la implementación de mejores prácticas de Gobierno Abierto en sus compromisos de reformas ${ }^{1}$. Los diferentes países que conforman la red, generaron una declaración donde puntualizan los siguientes elementos: 1) aumentar la disponibilidad de información sobre

1 Los países que firmaron la declaración el 20 de septiembre de 2011 fueron Brasil, Filipinas, Indonesia, México, Noruega, Reino Unido, Sudáfrica y Estados Unidos. Los países que han aprobado la declaración al 17 de abril de 2012 son Albania, Armenia, Bulgaria, Canadá, Chile, Colombia, Croacia, Dinamarca, Eslovaquia, España, Estonia, Georgia, Grecia, Guatemala, Honduras, Israel, Italia, Malta, Moldavia, Montenegro, Países Bajos, Perú, República Dominicana, Rumania, Ucrania y Uruguay. Y los países que están en proceso de aprobar la declaración son Azerbaiyán, Corea del Sur, Costa Rica, El Salvador, Ghana, Jordania, Kenia, Letonia, Liberia, Lituania, Macedonia, Mongolia, Panamá, Paraguay, República Checa, Rusia, Serbia, Suecia, Tanzania, Trinidad y Tobago y Turquía (http://www.opengovpartnership.org/ open-government-declaration) [26-02-2013]. 
las actividades gubernamentales; 2) apoyar la participación ciudadana; 3) aplicar los más altos estándares de integridad profesional en todos los gobiernos; y 4) aumentar el acceso a las nuevas tecnologías para la apertura y la rendición de cuentas. Así, los gobiernos signatarios se comprometieron en adoptar estos principios en su compromiso internacional, y trabajar para fomentar una cultura mundial de Gobierno Abierto que empodere a los ciudadanos y les cumpla, y avance los ideales de un Gobierno Abierto y participativo en el siglo XXI.

De esta manera, pues, transitar a un Gobierno Abierto implica un conjunto de cambios en la perspectiva de la cultura organizacional (sobre todo en la organización pública) y de gestión. César Calderón (2012), citando el blog de Javier Linares, presenta algunos cambios que deben realizarse en la implementación de un Gobierno Abierto:

1. Cambio en los procesos. Los procesos en la administración pública no han sido diseńados para servir a los ciudadanos, no son cómodos para el ciudadano o no le ayudan, y por lo tanto hay que re-ingeniarlos para conseguir que así sea, hay que eliminarlos o cambiarlos.

2. Cambio en la organización. Las organizaciones públicas están diseñadas bajo modelos jerárquicos que nada tienen que ver con la eficiencia. Es imprescindible reorganizar las administraciones, las plantillas y la definición de los puestos de trabajo para poder actuar bajo un modelo en red, orientado a proyectos y a la consecución de resultados.

3. Cambio en las formas de relación. Del mostrador a la mesa redonda, del correo certificado a la comunicación en línea, de la obligación a la presencia física a las facilidades de relación, etc. (2012: 27).

Otra visión es la de Mariñez Navarro y Valenzuela Mendoza (2013), quienes asumen que el Gobierno Abierto debe traducirse en un modelo de gestión, así como en una nueva forma de concebir la relación de colaboración gobierno-gobierno, gobierno-sector privado y gobierno-ciudadanos, y de esta manera convertirse en una plataforma para una democracia abierta. Para ello, proponen cuatro componentes incorporados en dos ejes. El primer eje se refiere al componente transversal: la transparencia; y el segundo eje lo componen la participación colaborativa, expresada en la relación ciudadanos-gobierno, la colaboración intra-intergubernamental y la asociación colaborativa público-privada como esquemas de gestión con sus respectivos criterios de rendimientos. 
Vemos entonces que en la actualidad, bajo los ejes de transparencia, participación y colaboración, el Gobierno Abierto se ha posicionado como una nueva forma de fortalecimiento de las capacidades del gobierno y la modernización de la administración pública, tomando exclusivamente como base las tecnologías de información ${ }^{2}$.

\section{DEL VOTO A LA PLATAFORMA ABIERTA DEL GOBIERNO}

Pero, no podemos situarnos en la perspectiva de un Gobierno Abierto sino vemos la convivencia democrática como un proceso participativo. Es decir, nos referimos a ir más allá del voto o de la democracia representativa o democracia procedimental. Ya hoy los gobiernos deben concebirse como plataformas de democracias participativas que tengan como características la transparencia, la participación ciudadana, la rendición de cuentas, la colaboración entre los ciudadanos y los gobiernos apoyados por las tecnologías de la información ${ }^{3}$ y, por supuesto, modelos de gestión con confianza que impliquen funcionarios responsables con ética pública ${ }^{4}$. Tim O’Reilly (2010) explica que el gobierno debe convertirse en plataforma de la democracia en esta nueva era de información.

Hay un nuevo pacto en el horizonte: información producida por y en nombre de los ciudadanos en la savia de la economía y la nación; el gobierno tiene la responsabilidad de tratar la información como un activo nacional. Los ciudadanos están conectados como nunca antes y tienen el conjunto de habilidades y la pasión para resolver problemas que les afectan

2 Se pueden destacar las investigaciones y propuestas empíricas de los siguientes autores e instituciones tales como Albert J. Meijer (2012), Teresa Harrison et al. (2011), Sharon Dawes (2010), Beth Noveck (2010), César Calderón y Sebastían Lorenzo (2010) y Daniel Lathrop y Laurel Ruma (2010), el Center for Tecnhology in Government de la Universidad de Albany y el Open Government Working Group del Gobierno de los Estados Unidos de América.

3 Después de la llegada de la World Wide Web, muchos anticiparon que el Internet podría revolucionar al gobierno, permitiendo un aumento en la participación política: una democracia electrónica, así como una revolución de e-commerce. Expertos anunciaron una nueva edad de Oro de Pericles y celebraban las oportunidades cívicas de las nuevas comunicaciones y tecnologías de la información. El ideal deliberativo de la gente con distintos orígenes y diferentes puntos de vista debatiendo y votando incluso sobre cuestiones públicas estaba a punto de convertirse en una realidad (Noveck, 2010).

4 El significado de la confianza en una gobernanza efectiva sugiere que el desarrollo de un modelo de confianza pública sea oportuna y práctica. El supuesto del desarrollo del modelo de confianza refleja una creencia de un parsimonioso modelo con un manejable número de factores que podría proveer bases sólidas para el estudio empírico de la confianza en las agencias del gobierno (Mayer, Davis y Schoorman, citado en Kim 2005: 614). 
localmente, así como a nivel nacional. Los servicios e información del gobierno se pueden proveer siempre a los ciudadanos cuando y donde lo necesiten. Los ciudadanos están facultados para encender la chispa de la innovación que dará como resultado un mejor enfoque a la gobernanza. En este modelo, el gobierno es un coordinador y un activador en lugar de mover primero la acción cívica (2010: 12-13).

Douglas Schuler (2010) deja claro cuatro conceptos que son la base y el centro de lo que sería una plataforma de democracia desde el gobierno. El primer concepto que este autor propone es la inteligencia cívica vista como inteligencia colectiva directa dirigida a enfrentar los desafíos compartidos. Su presencia o ausencia determinará cómo efectivamente estos desafíos pueden enfrentarse.

A causa de que el gobierno y otras elites grupales no son capaces de hacer frente a los problemas, se requerirá una forma más profunda de inteligencia cívica construida por ricas interacciones entre ciudadanos distribuidos en todo el mundo. Esta inteligencia no emergerá únicamente de una serie de votos u otras técnicas algorítmicas [...] (2010: 92).

El segundo concepto lo relaciona el autor con la democracia. Entendida como un ideal, ésta no es más que la forma de organización política que más estrechamente encarna la inteligencia cívica. En otras palabras es la intercepción entre el ideal y la práctica convivencial. Así, “[...] democracia se define también por procesos inclusivos y transparentes, aunque a veces se bloquea el acceso a estos procesos y además, estos a su vez son a menudo amañados por la élite política o económica" (2010: 92). El tercer concepto propuesto por Schuler es el Gobierno Abierto, que ofrece un grupo provocativo de ideas para la reconstrucción del gobierno con el objetivo de aumentar y mejorar las habilidades de las sociedades democráticas para enfrentarse efectivamente, sustentablemente y equitativamente a cuestiones o problemas.

Y el cuarto concepto es la deliberación en línea (online deliberation), que es vista como un proceso de comunicación directa, a través de las tecnologías de la información disponibles, mediante la cual la gente discute de manera abierta sus razones, intereses, habilidades y valores, con la intención de llegar a un acuerdo para la toma de decisión. Lo más importante es que al llegar a una decisión, ésta sirva de marco o plan para compartir con un grueso número de gente. La capacidad en la deliberación es importante para el esquema más general de la capacidad de la inteligencia cívica. Para Schuler, la deliberación se concibe: 
[...] distinta de otras modalidades de comunicación tales como la reflexión individual, la repetición y los múltiples esfuerzos por compartir enfoques, adquiriendo puntos de vistas solamente a través de exposiciones de los medios de comunicación, o trabajando para derrotar una persona, idea o empresa, no a través de los meritos de un propio argumento o la falta de meritos de la otra, sino por cualquier otro medio necesario (no violentos), incluyendo la difamación y la mentira (2010: 93).

En este sentido, Lori A. Brainard y John G. McNutt (2010) complementan a Schuler cuando explican un nuevo modelo de gestión, denominado Nuevo Servicio Público para diferenciarlo de la administración pública weberiana y de la Nueva Gestión Pública. Aunque estos dos últimos modelos de gestión se sustentan en la teoría de la elección pública y la teoría de la elección racional, respectivamente, las dos propuestas divergen ya que en una administración pública weberiana la ley encarna el interés público, que se deriva del área de la política (Poder legislativo), aquí el Estado es el único encargado de proveer con eficiencia y efectividad los bienes, la información y los servicios en una relación autoritaria regulada con los ciudadanos y grupos voluntarios. Y la Nueva Gestión Pública visualiza el interés público como resultado de la búsqueda de los intereses privados donde el Estado actuaría como facilitador de las elecciones individuales usando técnicas del mercado. Aquí las relación entre el gobierno y, los ciudadanos y organizaciones voluntarias es transaccional orientada al intercambio.

Por otro lado, el Nuevo Servicio Público parte de la premisa de las motivaciones democráticas para participar en la esfera pública. De esta manera, concibe el interés público como resultado del diálogo. Se visualiza a la gente como ciudadanos y el rol primario del funcionario público es servir y facilitar la participación de y en colaboración con los ciudadanos antes que un funcionario que meramente implementa programas preconcebidos para clientes o como una vía catalizadora para las fuerzas del mercado.

El enfoque con énfasis en asociaciones de base y compromiso ciudadano en la vida pública es parte de una tendencia mayor dentro de los académicos de la administración pública ya que replantea el papel del administrador público como un experto neutral a diferencia de un facilitador de los ciudadanos con bases de participación y compromiso. En este escenario, los administradores públicos crean oportunidades para el compromiso con los ciudadanos, utilizando esas oportunidades para educar, organizar y activar a estos para promover y participar en la esfera pública en su propia representación. También significa que los administradores públicos se comprometerían con los ciudadanos a colaborar para identificar y definir 
los problemas, y crear y aplicar sus soluciones. Como parte de esto, las relaciones gobierno-ciudadanos se convertirían en más deliberativas y dialógicas antes que regulativas y basadas en la autoridad" (Brainard y McNutt 2010: 841).

El Gobierno Abierto, entonces, no lo podemos explicar sin mecanismos institucionales sólidos que permitan a una democracia hacer de lo público lo que es del público por lo que las decisiones públicas deben someterse al escrutinio de los ciudadanos, es decir, en el marco de un nivel de transparencia donde el gobierno esté dispuesto a permitir a la gente monitorear sus resultados y a participar en sus procesos de política pública. También implica que los funcionarios públicos y los políticos estén sometidos día a día a la rendición de cuentas tanto horizontales como verticales (representación y societal) que la participación de los ciudadanos, las asociaciones público-privados y las relaciones intra e intergubernamental estén marcados por la colaboración deliberativa para que la política pública y los servicios públicos, además de ser de calidad, sean respuestas a las preferencias y derechos de la gente.

De esta manera, tal como lo precisa Cusba (2012), la colaboración es entonces uno de los elementos que forman parte de ese gran cambio que se produce en las formas de gestionar los intereses públicos. Tradicionalmente, los ciudadanos han colaborado a través de mecanismos diferentes, unos cooptados y otros más espontáneos. Pero la colaboración vista a la luz del Gobierno Abierto ofrece a los ciudadanos la posibilidad de compartir lo que saben y sobre todo de crear soluciones que generan valor en donde realmente quieren y pueden aportar. No quiere decir esto que el gobierno -como institución legitimada y legitimadora políticamente- deje de ser el centro de la toma de decisiones, por el contrario, además también se beneficia de las contribuciones de los que están fuera de él. En otras palabras, la colaboración ofrece una posibilidad potencial para construir de forma más eficaz y legítima los gobiernos por la vía de la transparencia y la rendición de cuentas.

\section{TRANSPARENCIA AL DÍA DE HOY: ¿NUEVAS PROPUESTAS?}

Hoy se están encontrando nuevas pistas a la definición de la transparencia, por lo que nos preguntamos ¿qué transparencia necesita el Gobierno Abierto? La apertura del flujo de información, tal como se le define, en el marco de la relación entre gobierno y ciudadanos nos sumerge al concepto de la nueva transparencia, que explica cómo las organizaciones públicas y actores gubernamentales se enfrentan cada vez más a las demandas de la sociedad para revelar información. Es decir, forzar al gobierno y al sector cívico al movimiento por una sociedad abierta. Ya no es el tiempo en que los 
gobiernos pasivamente proveían información solamente sobre lo solicitado y a la discreción gubernamental, sino el requisito de más compromiso activo en la información pública, comunicar las operaciones internas de la gestión. Por eso la relación entre transparencia, información y democracia es fundamental y básica. La información porque ayuda a las competencias democráticas como la formulación de preferencias y opiniones probadas, y la participación, porque provee elementos significativos en la hechura de la decisión pública. Archon Fung y David Weil (2010), explican con respecto a la transparencia que se deberían tomar en cuenta dos principales escollos que podrían estropear lo anteriormente explicado:

El primero es que esta transparencia gubernamental, aunque impulsada por intenciones progresistas, podría desviar una atención excesiva hacia los errores del gobierno y, por lo tanto, tendrían la consecuencia de reforzar una imagen conservadora del gobierno como incompetente y corrupto. El segundo es que toda esta energía dedicada a hacer un gobierno abierto tiene el costo de dejar las operaciones de grandes organizaciones del sector privado, como bancos, fabricantes, proveedores de servicios salud, productores de alimentos, compañías farmacéuticas y similares, de forma opaca y secreta (2010: 106).

El acceso a la información pública está tomando un énfasis renovado en todo el mundo, reflejándose a través de las siguientes tendencias: 1) a las visiones de la reforma gubernamental que presiona la apertura y la transparencia; y 2) al aumento de la disponibilidad y desarrollo continuo de las TICs que permite a las instituciones, organizaciones e individuos buscar de la misma forma, así como compartir, combinar y reusar el contenido de la información del gobierno ${ }^{5}$.

Para Albert J. Meijer (2012), en esta época de la transparencia gubernamental (government transparency), uno de los temas fundamentales a ser abordados son la relación conceptual entre la transparencia del gobierno y la participación ciudadana ${ }^{6}$; la naturaleza y los efectos de los

5 La información TICs para informar al público emerge en los ańos 90s con el crecimiento del e-government, y ello ha contribuido a la transparencia gubernamental. Hoy, la tendencia es ver secretarías, departamentos y agencias gubernamentales poniendo internamente grupos de datos electrónicos para el uso del público sobre nuevas government-wide website, así como Data.gov, elemento esencial del Gobierno Abierto diseñado para facilitar el acceso al grupo de datos a nivel del gobierno federal con el objetivo de aumentar la comprensión pública de las operaciones, avances de la misión gubernamental, creación de oportunidades económicas y un aumento de la transparencia, rendición de cuentas y responsabilización.

6 Mucha de la discusión sobre la transparencia del gobierno se focaliza sobre la relación entre la disponibilidad de la información (government transparency) y el uso de 
mecanismos de la transparencia fiscal ${ }^{7}$; el efecto de la transparencia en la confianza del gobierno; el efecto de la transparencia interinstitucional en el control parlamentario ${ }^{8}$; y la relación empírica entre la transparencia del gobierno y la participación ciudadana. Estos temas relacionados con la transparencia del gobierno llevan al mismo autor a preguntarse lo siguiente: ¿la transparencia es creada por presiones de afuera (ciudadanos, stakeholders, medios de comunicación) o por tensiones internas de los sistemas gubernamentales? ¿Cuáles son los sectores que se han hecho más transparentes? ¿Cómo usan la transparencia los ciudadanos, stakeholders y los medios de comunicación? ¿Cambia la transparencia el comportamiento de los funcionarios públicos y de las organizaciones públicas?

Muchos autores, como Piotrowski (2007), han afirmado que este tipo de transparencia es fundamental para el Gobierno Abierto. Y por otro lado, Sharon S. Dawes (2010) argumenta que las políticas públicas relacionadas con el flujo de información son uno de los aspectos más importantes de la democracia ya que refleja un tipo de elección societal en el que se estimaría cómo la información debería producirse, procesarse, guardarse, intercambiarse y regularse. Entendida así, la transparencia se está constituyendo en una importante agenda tanto en las organizaciones públicas como privadas. Viéndose así entonces, la transparencia pudiera definirse como la disponibilidad de información para el público general y la claridad sobre las reglas, regulaciones y decisiones del gobierno. Es decir, al plantearlo con más precisión, la transparencia refiere entonces a la disponibilidad y aumento del flujo de información oportuna, clara, relevante, de alta calidad y confiable, relacionada con las actividades de la organización gubernamental que por supuesto impacta a la gestión pública misma.

No obstante estas premisas de la transparencia gubernamental, Karr (citado en Dawes 2010), sintetiza tres tipos de tensiones asociadas con el uso público de la información gubernamental. La primera tensión es la

esta información por ciudadanos y stakeholders (participación). Transparencia del gobierno y participación son dos avenidas del Gobierno Abierto (Meijer 2012: 5).

7 David Heald argumenta que el significado específico de transparencia fiscal puede definirse en cuatro términos: hacia adentro, hacia afuera, hacia arriba y hacia abajo. El artículo muestra que hay barreras intrínsecas para la transparencia sobre el gasto público tales como las complejidades técnicas y el "lenguaje” de medición que también construyen barreras creadas para proteger a intereses específicos. Se concluye que la transparencia sobre el gasto público no puede proporcionar respuestas a cuestiones ideológicas, pero sí puede mejorar la base de evidencia para el debate público (citado en Meijer 2012: 6).

8 Mientras que la transparencia refiere a la disponibilidad de la información para el público general, la transparencia interinstitucional es referida a la transparencia entre las instituciones del gobierno (Meijer 2012: 6). 
que se presenta entre la amplitud de los datos y su comprensión por los ciudadanos no preparados técnicamente; la segunda tensión se da entre los deseos para apoyar la utilidad detallada de los datos y, simultáneamente, proteger la confidencialidad de los temas de los mismos; y la tercera y última tensión se visualiza cuando el público necesita y desea analizar y comprender un grupo de datos globales, mientras que en la realidad los datos del gobierno están fragmentados y distribuidos a todos los niveles del gobierno a través de distintas organizaciones y ámbitos en que se genera y desarrolla la política pública.

Ball (citado en Park y Blenkinsopp 2011) sugiere que la transparencia está empezando a combinarse con la rendición de cuentas en el discurso público sobre la gobernanza democrática. Ello ha permitido verla en base a varios factores: primero, la transparencia es uno de los fundamentos morales que sostienen las sociedades democráticas, a través de los derechos de los ciudadanos al acceso de la información gubernamental; segundo, la transparencia es una de las medidas prácticas tomadas para restringir la corrupción, actuar como una fuerza contra el comportamiento corrupto de la burocracia, promoviendo la vigilancia de los ciudadanos para evitar el rentseeking; y tercero, la transparencia tiene un efecto positivo en la confianza y en la rendición de cuentas con la idea que los ciudadanos monitoreen la calidad de los servicios públicos y motiven a los funcionarios públicos a satisfacer los ciudadanos, permitiendo aumentar el grado de confianza ya que ello es un importante principio de la democracia deliberativa 9 Estos tres factores nos abren la posibilidad de visualizar tres componentes esenciales cuando pensamos en la transparencia gubernamental: el acceso a la información pública como derecho ciudadano ${ }^{10}$, una burocracia con

9 En el caso mexicano, con la carencia de sanciones que tiene el instrumento legal de la transparencia, la rendición de cuentas se ve más imposibilitada ya que padece de una fragmentación institucional. De esta manera, mientras en el país no se tenga claro que la accesibilidad y exigencia de la información pública forma parte del derecho (a informar) y por ende, que es una política pública que expresa este derecho, esta práctica democrática se verá limitada (López Ayllón, Merino y Morales 2011).

10 El Centro Carter visualiza el acceso a la información como un derecho humano fundamental en todas las culturas y sistemas de gobierno. En este sentido, ha establecido un plan de acción para asegurar estos derechos: 1) ejercicio equitativo del derecho de acceso; 2) preparación y formación de los funcionarios públicos en la práctica y aplicación de los derechos de acceso; 3) educación pública para autorizar el uso completo del derecho; 4) asignación de recursos necesarios para asegurar una administración eficiente y oportuna; 5) intensificación de la gestión de la información para facilitar el acceso a la información; 6) práctica habitual en el monitoreo y evaluación; y 7) supervisión de operación y acatamiento (Carter Center, citado en Dawes, 2010). 
ética pública ${ }^{11}$, y la confianza institucional ${ }^{12}$.

Según Papenful y Schaefer (2010), tres pasos deben ser completados para lograr una rendición de cuentas pública. El primer paso, el acceso a la información, garantizada y completa, así como no distorsionada ni atrasada y sin altos costos. Ello permitirá minimizar problemas de asimetría de la información ${ }^{13}$. El segundo, la calidad de la información, debe estar asegurada a través de los informes de cooperación (reports). Estos no son más que un tipo de información sumaria agregada con detalles individuales donde se explican los elementos clave que pueden ser comprendidos por los ciudadanos interesados. Los informes de cooperación incluyen un mapa organizacional de la estructura de la autoridad pública que tiene como objetivo proveer las bases del cómo se deciden los problemas públicos para garantizar una provisión de servicios públicos sustentables, eficaces y eficientes. Con el tercer paso, se debe garantizar al ciudadano su capacidad de explicación, una información no distorsionada y al día y en el momento.

Hoy es importante, al sistematizar las bases del Gobierno Abierto, replantear la transparencia en la gestión pública para que el carácter multifacético y diverso de la provisión de los servicios públicos, la ejecución de servicios, el financiamiento y la administración misma minimicen lagunas de opacidad, generando que la relación entre ciudadanos y agentes (políticos, administración y gerentes públicos) sea la más apropiada democráticamente hablando.

La entrega y facilitación del acceso a la información pública es un aspecto central para la efectividad de los gobiernos. El análisis de resultados sobre el desempeño de la gestión pública puede retroalimentar, entre otros aspectos: i) el rediseño de procesos administrativos; ii) el abaratamiento de los costos y la aceleración del tiempo de las transacciones, y iii) el mejoramiento de la rapidez y

11 Los funcionarios públicos que no visualicen el acceso a la información pública como derecho corren el riesgo de implementar una gestión opaca y carente de una ética pública.

12 Kasperson et al. (citados por Van der Meer 2010) argumenta que la confianza es una evaluación relacional de situaciones sociales a través de cuatro dimensiones: Una persona que confía (el sujeto); da a entender que él o ella piensan que el objeto de confianza reúne ciertos requerimientos: el objeto sería competente, tanto intrísecamente (cuidado) como extrínsecamente comprometido (auditable a causa de su interés concreto) y predecible.

13 Es evidente que la relevancia de la información depende de quiénes sean los usuarios potenciales de la información. Sin embargo, hay una primera delimitación que puede hacerse cuando estamos hablando de transparencia: en este caso, la referencia obligada es la ciudadanía, de manera que la relevancia de la información estará condicionada a si sirve o no a sus fines (Cunill Grau, 2006) 
calidad de los servicios a los ciudadanos (Baena Olabe y Cruz Vieyra 2011: 25).

En esta dirección, es importante destacar que el Open Government Working Group recomienda ocho principios para el acceso y uso de los datos públicos, con el objetivo de promover el discurso cívico, el bienestar público y más eficiencia en el uso de los recursos públicos:

1. Todos los datos públicos deben estar disponibles, exceptuando los que tocan la privacidad y la seguridad (información completa).

2. Los datos son recolectados en la fuente primaria, con un nivel de sutilidad posible fragmentada (datos primarios).

3. La disponibilidad de los datos debe estar disponible tan rápido como sea necesario para preservar el valor de los mismos. (información oportuna).

4. La información está disponible para el más amplio número de usuarios y de objetivos (accesibilidad de datos).

5. La estructuración de los datos debe ser razonable para permitir su procesamiento automático (procesamiento de la información).

6. Los ciudadanos deben disponer de los datos sin requerimientos de registros (acceso sin discriminación).

7. La disponibilidad de los datos en un formato en la cual ninguna entidad tiene un control exclusivo. Los datos son bienes públicos (la no propiedad de la información).

8. Los datos no están sujetos a licencias (copyrigth), patentes, marcas comerciales o secretos de regulación comercial. Sólo por razones de privacidad, de seguridad y de restricciones de privilegios debe ser permitida su secrecía (información libre de licencia).

El concepto de accountability, también usado como rendición de cuentas en el sector público, debe ser usado en consecuencia para reportar el uso de recursos por parte del gobierno, así como el rendimiento de los servicios. Para ello la transparencia es un elemento fundamental ya que es una herramienta por lo que es observado el proceso de la hechura de la decisión pública. Mientras que la participación refiere a la oportunidad de participar en ese proceso. O como Curtin y Mendes lo afirman (citado en Meijer, Curtin y Hillebrandt 2012), "los ciudadanos necesitan información para ver qué se está haciendo al interior del gobierno, y participación para expresar sus opiniones sobre el quehacer gubernamental" (2012: 11). De esta manera, los ciudadanos no solamente tienen acceso a la información, 
sino que se convierten en participantes con expresión activa. Así, la elección de los usuarios como sus opiniones con respecto a los servicios públicos son fundamentales para la legitimidad del gobierno.

Recientemente ha habido un énfasis fuerte en la política pública en escuchar a los ciudadanos y a los usuarios de los servicios a través de choice y voice. PASC (citado en Simmons, Birchall y Prout 2011) define la elección (choice):

[...] como preferencias individuales que da la oportunidad de elegir de entre las alternativas ofrecidas, enteramente o no del sector público. Expresión (voice) es definida como las preferencias más efectivas expresadas por los usuarios de los servicios a través de cuerpos representativos, mecanismos de reclamos y encuestas de opiniones de preferencias individuales (2011: 4).

En esta perspectiva, entonces, la transparencia está discutiéndose hoy por hoy como una solución instrumental a problemas de legitimidad y confianza institucional (Kim 2005). Ello no sería posible sin una participación colaborativa entre los actores gubernamentales y no gubernamentales, ciudadanos y organizaciones cívicas.

\section{LA CONSTRUCCIÓN DE UN NUEVO CONCEPTO DE TRANSPARENCIA}

El acceso a la información pública está tomando un énfasis renovado en todo el mundo, reflejándose a través de las siguientes tendencias: 1) las visiones de la reforma gubernamental que presiona la apertura y la transparencia; y 2) el aumento de la disponibilidad y desarrollo continuo de las TICs que permiten a las instituciones, organizaciones e individuos buscar de la misma forma, así como compartir, combinar y reusar el contenido de la información del gobierno. De esta forma la transparencia puede ser usada como instrumento de políticas públicas (Fung, Graham y Weil 2007), implicando que la información debería producirse, procesarse, guardarse, intercambiarse y regularse. De esta manera, podríamos establecer la diferencia entre la transparencia opaca y la transparencia clara (Fox 2008). 


\section{Tabla 1: Transparencia opaca y transparencia clara}

Transparencia opaca

(Gobierno democrático sin plataforma de Gobierno Abierto)

- Formas de difusión de información oficial que no revelan cómo se desempeñan las instituciones en la práctica, ya sea en términos de cómo se toman decisiones o de cuáles son los resultados de sus acciones.

- No vinculada con la rendición de cuentas

- Se concibe políticamente vinculada a los derechos de acceso a la información.

- Los actores interesados (usuarios) no alcanzan a tener estrategias de cambio constructivo para la institución.

\section{Transparencia clara}

(Gobierno democrático con plataforma de Gobierno Abierto)

Se refiere a programas que dan a conocer información confiable y accesible sobre el desempeño institucional, precisando las responsabilidades de sus funcionarios, particularmente en lo relativo a la toma de decisiones, así como el destino real de sus recursos.

- Se vincula con la rendición de cuentas a través del siguiente itinerario:

- Acceso a la información

- Calidad de la información

- Transparencia

- Se concibe como derecho, pero además como solución instrumental a problemas de legitimidad y confianza institucional.

Expresa una capacidad institucional de tal modo que permite que los actores interesados puedan anticipar estrategias de cambio constructivo para la institución

Fuente: Elaboración propia en base a Fox (2008).

Por otro lado, la maduración de las políticas de acceso a la información han generado un debate sobre cómo puede lograr una mayor efectividad y un beneficio más amplio para la sociedad derivado de la transparencia. De esta manera, existen dos enfoques. El primero es el más general, relacionado con la sanción y puesta en práctica de leyes de acceso a la información que logre un objetivo principal, tal es: 
[...] incorporar los principios o estándares reconocidos internacionalmente en el ordenamiento legal de los países. Estos principios incluyen, entre otros: la máxima apertura de la información, la definición del conjunto de excepciones que limita el acceso a la información en casos puntuales, la proactividad en la publicación de información por parte de las entidades estatales sin esperar requerimientos para hacerla accesible, y la efectiva implementación de las leyes, además del monitoreo de su cumplimiento (Baena Olabe y Cruz Vieyra 2011).

El segundo enfoque se vincula al diseño y a la implementación de sistemas o mecanismos en sectores o áreas de intervención específicas, independientemente de la existencia de una ley general de acceso a la información (enfoque sectorial o focalizado).

Por enfoque sectorial se entiende la promoción del acceso a la información a través del diseño y de la implementación de sistemas o mecanismos de acceso en sectores o áreas de intervención específicas, como la educación, la justicia, la elaboración, la ejecución y el control de los presupuestos públicos, el financiamiento de las campañas políticas, o las industrias extractivas, independientemente de la existencia de un marco normativo general de acceso a la información. Una de las características principales de este enfoque es el reconocimiento de que cada sector tiene vulnerabilidades y dinámicas de funcionamiento propias, las cuales determinan los contenidos y formas de intervención para promover el acceso a la información (Baena Olabe y Cruz Vieyra 2011: 9).

En este sentido, este enfoque, más que un principio, sería un dispositivo que hace que la política aumente la probabilidad de éxito.

Afortunadamente, las leyes y políticas que obligan a dar información de las empresas han surgido en varios ámbitos de la política en las últimas décadas. En otro trabajo, hemos denominado a estas medidas Transparencia focalizada, ya que no sólo tienen por objeto proporcionar información general, sino específica de públicos, como mejores escuelas, hospitales de alta calidad y más cuidado de los consumidores y productos (Fung, Graham y Weil 2007: 109).

La primera generación de la transparencia se caracterizaba por la apertura general de toda la información del gobierno a todos los ciudadanos para cumplir con un derecho político fundamental. Hoy esa apertura debe focalizarse, de tal forma que determinados procesos, trámites, así como sectores sociales específicos puedan mejorar y beneficiarse de la 
transparencia.

La transparencia focalizada es una respuesta a la necesidad de obtener beneficios específicos de la información, así como a la demanda de un mayor orden en la información que ofrece el gobierno. Este tipo de transparencia requiere que el gobierno estandarice y publique información específica, que busque resolver un problema social concreto, y estimule los mecanismos del mercado para lograr un beneficio social específico.

Las políticas de transparencia focalizada implican que: 1) el centro de atención es el usuario de la información (sus necesidades, intereses y capacidades para entender la información); y 2) están basadas en las necesidades, intereses y capacidades de publicación de las organizaciones civiles, públicas y corporaciones. Así, su principal objetivo es el de influenciar la capacidad de toma de decisiones de los usuarios de la información (Fung, Graham y Weil 2007). En esta definición lo que se sostiene es que un actor (casi siempre el gobierno) le indica a otro actor (una corporación, empresa etc.) que divulgue información con un propósito específico para que la gente la utilice.

De esta manera, es el Estado quien, en última instancia, decide la legitimidad y credibilidad de la provisión de la información. La transparencia focalizada se caracterizaría entonces por la divulgación pública obligatoria, dirigida a corporaciones $\mathrm{u}$ otras organizaciones privadas o públicas, información estandarizada, comparable y fraccionada, con énfasis a productos o prácticas específicas y con un objetivo público claro. En la siguiente tabla establecemos la diferencia entre la transparencia general y la focalizada. 


\section{Tabla 2: Enfoques de la transparencia}

Enfoque general o comprensivo

- Foco exclusivo en el diseño, la implementación y el cumplimiento de leyes de acceso a la información a nivel nacional.

- Resultados en el largo plazo, cambio cultural.

- Basado en la creación de nueva legislación a nivel nacional.

- Necesidad de un cuerpo de leyes existentes para ser implementado eficazmente.

- Necesidad de la coalición de varios grupos interesados para generar voluntad política suficiente.

- Los usuarios no perciben los objetivos de estas leyes como beneficios inmediatos o concretos.

- Los resultados son más difusos (cambio cultural) y de largo plazo.
Enfoque sectorial (focalizada)

Foco en mecanismos, sistemas o procedimientos que permitan el acceso a la información en distintas áreas y sectores del gobierno.

- Resultados en el corto plazo o mediano plazo, e impacto específico en los sectores críticos.

- $\quad$ Se construye a partir de la legislación existente.

- Mayor facilidad para identificar y aprovechar oportunidades de voluntad política.

- $\quad$ No se necesita de grandes coaliciones de intereses para poder implementar mecanismos o sistemas de acceso.

- Mayor facilidad de los usuarios para interpretar los beneficios concretos de la aplicación de las políticas o mecanismos.

- Mayor flexibilidad para medir resultados.

Fuente: Baena Olabe y Cruz Vieyra (2011: 9).

\section{LA TRANSPARENCIA COMO BIEN PÚBLICO Y COMO INTERÉS PÚBLICO}

Entendido así, la transparencia se está constituyendo, además del qué decir de las sociedades contemporáneas, en una importante agenda tanto de las organizaciones públicas como privadas. Se verá, entonces, que la disponibilidad de información para el público general y la claridad sobre las reglas, regulaciones y decisiones del gobierno, así como la disponibilidad y el aumento del flujo de información oportuna, clara, relevante, de alta calidad y confiable relacionada con las actividades de la organización gubernamental impacta por supuesto a la gestión pública misma. Ello implica que esta parte sea socializada en las rutinas en la hechura de las tomas de decisiones tanto por los usuarios como por los sujetos obligados establecidos en la ley.

Significa, entonces, que la transparencia, información producida, guardada, organizada y gerenciada por el Estado a través de los sistemas gubernamentales de gestión (financiera, de personal, contratación, 
auditoría, fiscalización, etc.), debe considerarse como bien público y como interés público. Esta distinción tiene una importancia clave en la transparencia contemporánea. Como bien público, se explica cuando el gobierno como organización pública produce este bien desde el momento en que le solicita al sector privado y/o al mismo sector gubernamental información demandada por los ciudadanos, empresas privadas $u$ organizaciones de ciudadanos (usuarios de la información). Y como interés público, cuando el gobierno provee la transparencia de la información como servicio público con la idea de lograr la publicidad que no es más que la información divulgada para los fines de que el ciudadano o sector tenga la oportunidad de conocer y exigir información de la hechura de las decisiones y de las políticas públicas ${ }^{14}$.

Al sistematizar las bases de la transparencia como interés público, se requiere replantear el concepto clásico de la misma en la gestión pública. Hoy no es sólo el mandato legal el eje del interés público, estamos en presencia de otros tipos de legitimidades que dan un sentido público a la transparencia, nos referimos a la vinculada a las nuevas configuraciones en la relación gobierno-ciudadanos. En esta misma dimensión, cuando hablamos de transparencia podemos sistematizar según las premisas de David Heald la distinción siguiente:

[...] si la transparencia tiene como expectativa dirigirse a combatir la corrupción o si el fin de la transparencia es el logro de la eficiencia y la eficacia del uso de los recursos públicos y/o aumentar la legitimidad y rendición de cuentas de las instituciones públicas (2012: 31).

\section{LA TRANSPARENCIA FOCALIZADA COMO POLÍTICA PÚBLICA}

La transparencia focalizada se caracteriza por la divulgación pública obligatoria, dirigida a corporaciones $\mathrm{u}$ otras organizaciones privadas o públicas, la información estandarizada, comparable y fraccionada, con énfasis a productos o prácticas específicas y con un objetivo público claro. Así, entonces, con este concepto, el gobierno en su rol regulatorio, es un recolector, productor, proveedor y utilizador de la información.

En este rol, el gobierno trata la información como un instrumento de política. Al hacerlo, toma decisiones sobre sí y cómo recoger,

14 También la información producida, guardada y organizada por las empresas privadas es considerada información pública, dado al uso por los gobiernos y ciudadanos. Y el acceso a esta información pública -documentos, archivos, estadísticas-constituye uno de los fundamentos para la consolidación de la democracia y la apertura gubernamental, además de fortalecer las capacidades de los ciudadanos para participar de modo efectivo en las decisiones que les afecten, además de que le da mejores condiciones al ciudadano para conocer y acceder a otros derechos esenciales. 
elaborar, difundir, analizar y preservar la información al servicio de algún principio de otra política (como la salud pública, la calidad del medio ambiente, o desarrollo económico. (Dawes 2010: 1).

En este sentido, la elaboración, diseño e implementación de la política de transparencia focalizada tendría que establecerse con el mayor detalle posible. Archon, Graham y Well (2007), dejan ver muy claro dos momentos en la política. Primero, los sistemas de transparencia mejoran cuando enfocan sus recursos a lograr mayor precisión, calidad y utilidad de las aperturas. Existen varios factores que impulsan a los sujetos obligados a empujar en el tiempo mejoras en la transparencia. Segundo, usuarios dispersos de información podrían integrar agrupaciones o coaliciones que mejoraría la manera de exigir una mejor apertura.

En base a estos momentos de la política propuestos por Archon, Graham y Well (2007), proponemos un ejercicio de cómo una política de transparencia focalizada puede generar beneficios, ser sustentable y efectiva. Basta considerar la complejidad de la información y de los problemas que hacen que estos se vean con perspectiva más de incertidumbre. Hoy las políticas públicas podrían mirarse como desafíos a través de la perspectiva de la complejidad, capturando la realidad compleja diaria en la hechura e implementación de la política, caracterizada por lo que Robert Geyer y Samir Rihani (2010) denominan las expectativas de los actores centrales de la política. En este caso, nos referimos a las posibilidades de conseguir sus objetivos tanto los que provienen de la oferta -sujetos obligados de la transparencia-, como los usuarios de la información (demanda). En consecuencia, los actores de la política pública de transparencia deben continuamente flexibilizar la combinación de sus expectativas y entusiasmarse en el proceso de la política para adaptar y ajustar los cambios continuos en sus áreas.

Así, con estos dos momentos presentados por estos Geyer y Rihani (2010) para la formulación e implementación de la política de transparencia, podemos precisar una premisa importante: el nivel de transparencia de una organización refiere a la disposición de permitir a los usuarios al monitoreo de su rendimiento y a participar en los procesos de sus políticas. Nos quedarían las siguientes interrogantes a solucionar: ¿cuáles serían las relaciones de intermediación de intereses en una política de transparencia en un Gobierno Abierto? ¿Cómo estaría sustentada la previsibilidad de la política? ¿Qué mecanismos institucionales se diseñarían para que los usuarios puedan entender los procesos en las estructuras de gestión, financiera, regulativa y distributiva? ¿Cuáles serían sus creencias valorativas? ¿Qué instrumentos de la política se usarían para la implementación? ¿Cuál sería el rol de los actores (usuarios) en el desarrollo de la hechura de las 
decisiones?

Como el flujo de información en la sociedad contemporánea es cada día más rápido y el acceso a ella es menos rápido que el flujo, la transparencia como política pública entonces, tendería a explicarse bajo la óptica del cambio de política (policy change). Según Michiel S. De Vries (2010), el cambio de política no debe depender sólo por el cambio en los problemas.

[El] policy change no es más que el cambio de ideas, premisas, prioridades y fines, junto con el cambio en el uso de instrumentos de políticas y el rol cambiante de los actores societales y de los policymakers en el proceso de la política (2010: 6).

Esto nos lleva a precisar la conexión entre las relaciones de intermediación de intereses con la estabilidad de la política. En una política pública de transparencia en que el gobierno se convertía en proveedor pasivo de información, el modelo de intermediación de intereses (pluralismo y neocorporativismo) jugó un papel importante en la estabilidad de la política pública ${ }^{15}$. Hoy eso ha cambiado, ya que, como lo dice Jan Kooiman (2004), los conceptos de diversidad, complejidad y dinamismo han sido marcos para las nuevas relaciones ya que han surgido actores en sistemas sociopolíticos con objetivos, intenciones y poderes diversos, han aparecido estructuras, interdependencias e interrelaciones en y entre los diferentes niveles gubernamentales y societales, y se han manifestado nuevas dinámicas propias en los sistemas socio-políticos, así como la aparición de problemas, nuevas oportunidades e (i)regularidades de los desarrollos de los sistemas societales, políticos, económicos y culturales.

Un elemento también a considerar al tratarse de una política de transparencia hoy es el relacionado con el diseño de la política misma. Resolver el dilema de si es un diseńo con razonamiento racional en su hechura o un modelo de razonamiento diferente al racional (postracional ${ }^{16}$. Este dilema es un punto clave que se vincula con la posición

15 En los inicios de la difusión del pluralismo, durante los años cincuenta, en la literatura politológica norteamericana, se desarrollaron diversos modelos de policy-making que integraban las relaciones entre burocracia, políticos y organizaciones de intereses. Con la denominación de iron triangle, elastic nets, etc., se elaboraron modelos de interacción que analizaban la combinación de las relaciones formales e informales dentro de las reglas - por ejemplo el Congreso, la administración y los lobbies-, así como la capacidad de exclusión e influencia de los actores que constituían estas redes. Por otra parte, la difusión de los modelos neocorporatistas en buena parte como reacción a la inadecuación de la aproximación pluralista a los sistemas políticos europeos, sirvió para popularizar una concepción alternativa de la articulación de intereses (Jordana 2007: 511).

16 Deborah Stone (2012), en su libro La Paradoja de la Política, deja ver que en las 
y oposición de ideas, argumentación y valores entre los actores (sujetos obligados, usuarios y árbitros). Como dice Giandomenico Majone:

Los elementos justificativos desempeñan un papel más importante aún en la elaboración de políticas. En política no basta nunca con la decisión, aunque sea una decisión correcta. Las decisiones deben ser legitimadas, aceptadas y puestas en práctica. Tras la elección viene el proceso de justificación, explicación y persuasión. También los gobernantes actúan a menudo impulsados por influencias externas o por la fuerza de sus convicciones personales. En tales casos se requieren argumentos posteriores a la decisión para dotarla de una base conceptual, para demostrar que encaja en el marco de la política existente, para aumentar el asentimiento, para descubrir nuevas implicaciones y para adelantarse a las críticas o contestarlas (2005: 67).

En la decisión de la hechura de la política también deben tomarse en cuenta dos perspectivas, una vinculada con la política pública en sí, y otra relacionada con los aspectos administrativos de la hechura e implementación de la política. De esta manera, podemos esquematizar tres tipos de orientaciones: políticas orientadas a resultados (con alcance de los fines a corto plazo y eficacia a largo plazo); políticas orientadas a procesos (con procedimientos democráticos y eficiencia); y políticas públicas orientadas a soluciones que satisfacen normas dadas (con argumentos justificativos $y$, decisiones legítimas, aceptadas y puestas en prácticas).

políticas públicas se presentan dos modelos de razonamiento en las decisiones. Por una parte, el modelo de razonamiento de decisión racional caracterizado por el proceso clásico de política pública: identificación de objetivos, identificación de los cursos alternativos de acción para alcanzar objetivos, previsión de las posibles consecuencias de cada alternativa, evaluación de las posibles consecuencias de cada alternativa y selección de la alternativa que maximice el logro de los objetivos. Y por otro lado, el modelo de razonamiento de decisión post-racional con los siguientes rasgos: el razonamiento involucra la hechura de metáforas y de categorías, la identificación de los cursos de acción es producto del debate público o el impulso emocional de la gente, la importancia del contexto, cada visión construye un adversario político diferente, los analistas y/o ciudadanos están en la posición de detectar procesos y relaciones causales, dando significado a las palabras, al contexto y a las acciones. 


\section{Tabla 3: Dilemas en la hechura de la política pública de transparencia}

\section{Perspectiva política Perspectiva administrativa}

$\begin{array}{ccc}\text { Orientada a resultados } & \begin{array}{c}\text { Logro de objetivos a corto } \\ \text { plazo }\end{array} & \text { Eficacia a largo plazo } \\ \text { Orientada a procesos } & \text { Procedimiento democrático } & \text { Eficiencia } \\ \begin{array}{c}\text { Orientada a soluciones } \\ \text { que satisface normas } \\ \text { dadas. }\end{array} & \text { Argumentos justificativos } & \begin{array}{c}\text { Decisiones legítimas, } \\ \text { aceptadas y puestas en } \\ \text { prácticas }\end{array}\end{array}$

Fuente: Elaboración propia a partir de De Vries (2010).

Si partimos del hecho que el flujo libre de la información no es más que el flujo libre de un bien público, entonces la transparencia es importante regularla para no caer en la irresponsabilidad pública. En este sentido, el resultado del rendimiento de la transparencia podría explicarse a través de la comprensibilidad y de la información oportuna (Grimmelikhuijsen 2012). La comprensibilidad logra hacer a la transparencia más operativa para que la información divulgada sea más rápida y fácil de comprender. Y la información oportuna es caracterizada por una vigilancia continua. Esto implica que la "ventana de la transparencia" siempre debe estar abierta y que los sujetos obligados, la organización pública o privada, pueden ser vistos continuamente como responsables (accountable) (Heald 2012). Rawlins (2009): "llama a las organizaciones a compartir información inclusiva, auditable, completa, precisa, neutral, comparable, clara, oportuna, accesible, honesta y confiable, además de mantenerlas responsables" (citado en Birchall 2011: 14). Con esta reflexión varias interrogantes nos asaltan: ¿cómo se identifican los problemas públicos relacionados con la transparencia o acceso a la información desde los usuarios? ¿Cómo impactan los cambios tecnológicos y el uso de las TICs en la construcción de una agenda gubernamental de transparencia?, ¿En qué espacio público delibera la gente? ¿Cómo juegan los sujetos obligados, los usuarios y los árbitros?

\section{Las TeCNOLOGÍAS DE INFORMACIÓN Y COMUNICACIÓN Y LA TRANSPARENCIA}

Por otro lado, no podemos entender la transparencia en el Gobierno Abierto, si no revisamos las tendencias de los cambios tecnológicos en la gestión pública y la política pública. "La introducción de las TICs en la administración pública se puede considerar como una intervención social 
en una red de política que influyen la posición, los intereses, los valores y dominios (información) de los actores involucrados" (Homburg, citado en Pollitt 2011).

El cambio inducido (efectos) en la política y en la administración pública por el cambio tecnológico ha venido siendo estudiado por Christopher Pollitt (2011). En la perspectiva de los ciudadanos, este cambio ha tenido influencia en los lugares donde se ofrecen los servicios públicos, reconfigurándose estos a través de las nuevas tecnologías de transporte y comunicaciones tales como motorways, higher speed trains, video conferencing y más recientemente el high speed, y el intranets. Otro cambio, y tal vez el más significativo es el de los sistemas basados en web que hace que las relaciones ciudadanos-gobierno adquieran otra forma. Ya no es necesaria la presencia física del ciudadano en la oficina pública, a través de la relación virtual se pueden solicitar y ofrecer servicios. Pero también el espacio público de deliberación entre los ciudadanos (usuarios y solicitantes de información pública) ha cambiado en el momento en que se utilizan herramientas del Internet (Facebook, Twitter, etc.) para nuevos compromisos (online deliberation). De esta manera, en cualquier espacio y momento se podrían deliberar y discutir problemas públicos.

Una posición cyber-optimista es que las nuevas propiedades excepcionales del Internet motivan a todo tipo de gente para movilizar y participar a través de nuevos caminos en las cuestiones públicas. Una visión más cyberskeptic viene dado cuando los recursos en línea se utilizan principalmente para el refuerzo de los ciudadanos que ya están activos y muy bien comunicados por los canales tradicionales (Norris, citada en Pollit 2011: 385).

Así, el llamado de las redes sociales en los website es con el objetivo de poner las acciones sentidas minuto a minuto.

En cuanto a los cambios en los proveedores de servicios públicos es importante destacar que nuevas tareas son creadas mientras que otras son eliminadas. Por ejemplo, se mejoran los mecanismos institucionales de divulgación de la información pública, por lo que los funcionarios públicos adquieren nuevas habilidades y actitudes ante estos nuevos cambios, un ejemplo de ello son las formas de organizar las bases de datos, así como divulgar la información. Este elemento introducido por Pollitt (2011), al ejercitarlo con una política de acceso a la información, deja ver o nos aproxima a un tipo de transparencia del momento (real time). De este modo entonces, es importante destacar que sin ésta, y sin la rendición de cuentas, la democracia se convertiría en monarquía, totalitarismo o estructuras de poder oligárquicas que la democracia en sí las cataloga como 
contrarias, es decir, gobiernos con secrecía (Birchall 2011).

\section{CONCLUSIÓN}

Los avances de investigación sobre el Gobierno Abierto están profundizándose cada vez más. Ya los organismos internacionales lo han asumido en sus agendas tanto de investigación como de propuestas a sus gobiernos. Desde las visiones sustentadas por los estudios del Center for Tecnhology in Government de la Universidad de Albany, así como el Open Government Working Group, hasta las investigaciones académicas de Meijer (2012), Harrison et al. (2011), Dawes (2010), Noveck (2010), Calderón y Lorenzo (2010), entre otros, el concepto basado en los ejes como la transparencia, la participación y la colaboración ha sido una constante. En otras palabras, se dibuja una tendencia a una nueva relación gobierno-sociedad. Hoy el gobierno debe convertirse en plataforma de la democracia en esta nueva era de información. En consecuencia, el gobierno tiene la responsabilidad de tratar la información como un activo nacional y debe convertirse en un coordinador y activador de iniciativas de la gente ya que hoy más que nunca los ciudadanos están conectados y tienen el conjunto de habilidades y la pasión para resolver problemas que les afectan localmente, así como a niveles nacional e internacional.

Como lo hemos dejado ver en este artículo, sin transparencia y rendición de cuentas es imposible la colaboración y la participación de los actores no gubernamentales. Pudiera verse, entonces, la transparencia como la disponibilidad de información para un sector y la claridad sobre las reglas, regulaciones y decisiones del gobierno. Es decir, al plantearlo con más precisión, la transparencia refiere, entonces, a la eficiencia y eficacia de la gestión pública. Pero, ello también implica un conjunto de tensiones entre el gobierno y, los ciudadanos, stakeholders y medios de comunicación, como por ejemplo, las tensiones que se presentan entre la amplitud de los datos y su comprensión por los ciudadanos no preparados técnicamente; las que se manifiestan entre los deseos para apoyar la utilidad detallada de los datos y simultáneamente la que protege la confidencialidad de los temas de los mismos; y una última tensión, es aquella que se visualiza cuando el público necesita y desea analizar y comprender un grupo de datos globales mientras que en la realidad los datos del gobierno están fragmentados y distribuidos a todos los niveles del gobierno a través de distintas organizaciones y ámbitos en que se genera y desarrolla la política pública.

La anterior reflexión nos lleva a resolver la pregunta de si la transparencia es creada por presiones de afuera desde los ciudadanos y otros actores no gubernamentales, o si es producto de tensiones internas en el marco de 
las burocracias. Creemos que estas tensiones impulsan tanto a los actores gubernamentales como no gubernamentales a visualizar propuestas tendientes a minimizarlas. Otro cuestionamiento presentado es el cómo usan la transparencia los ciudadanos y otros actores no gubernamentales. Reflexionar esta interrogante nos lleva a aproximarnos a un esquema más analítico desde el punto de vista sustantivo y procedimental. En este sentido, la transparencia la tenemos que ver relacionada y combinada con la rendición de cuentas, la participación y el derecho de los ciudadanos a la información para permitir aumentar el grado de confianza entre gobierno y ciudadanos. Visto de esta manera, los tres pasos señalados en el transcurso del trabajo, para el logro de la rendición de cuentas (el acceso a la información, la calidad de la información y la transparencia) son los aspectos más importantes de la democracia en el momento que refleja un tipo de elección societal en el que se visualizaría cómo la información debería producirse, procesarse, guardarse, intercambiarse y regularse, tomando en cuenta la colaboración de los actores gubernamentales y no gubernamentales.

La transparencia vista como política pública en el Gobierno Abierto abre la posibilidad que el gobierno trate la información como un instrumento de política y se focalice a un sector específico. Al hacerlo, toma decisiones sobre si y cómo recoger, elaborar, difundir, analizar y preservar la información al servicio de algún principio de otra política (como la salud pública, la calidad del medio ambiente, o desarrollo económico). Así, podría verse como desafío a través de la perspectiva de la complejidad, capturando la realidad compleja diaria en la hechura e implementación de la política caracterizado por las expectativas de los actores centrales de la misma (sujetos obligados y los usuarios de la información). En consecuencia, los actores de la política pública de transparencia deben continuamente flexibilizar la combinación de sus expectativas y entusiasmarse en el proceso de la política para adaptar y ajustar los cambios continuos en sus áreas, porque el nivel de transparencia de una organización refiere a la disposición de permitir a los usuarios al monitoreo de su rendimiento y a participar en el proceso de sus políticas. 


\section{REFERENCIAS}

Baena Olabe, P. y Cruz Vieyra, J. (2011). Acceso a la Información y Políticas de Transparencia Focalizada. Washington: BID.

Birchall, C. (2011). Introduction to 'Secrecy and Transparency': The Politics of Opacity and Openness. Theory Culture \& Society, 28 (7-8), 7-25.

Brainard, L y McNutt, J. (2010). Virtual Government-Citizen Relations: Informational, Transactional or Collaborative? Administration \& Society, 42 (7), 836-858.

Calderón, C. y Lorenzo, S. (Coords.). (2010). Open Government. Gobierno Abierto. Jaén: Algón Editores.

Calderón, C. (2012). Por Qué un Gobierno Abierto. En Concha, G. y Nasser, A. (Coords.), El Desafío en el Gobierno Abierto en la Hora de la Igualdad. Santiago de Chile: CEPAL.

Cunill Grau, N. (2006). La Transparencia en la Gestión Pública. ¿Cómo Construirle Viabilidad? Estado, Gobierno y Gestión Pública. Revista Chilena de Administración Pública, 8, 22-44.

Cusba, E. (2012). Colaboración: El Gobierno en Doble Vía con las Personas. En Concha, G. y Nasser, A. (Coords.), El Desafio en el Gobierno Abierto en la Hora de la Igualdad. Santiago de Chile: CEPAL.

Dawes, S. (2010). Stewardship and Usefulness: Policy Principles for Information-Based Transparency. Government Information Quarterly, 27 (4), 377-383.

De Vries, M. (2010). The Importance of Neglect in Policy-Making. Londres: Palgrave Macmillan.

Fung, A. y Weil, D. (2010). Open Government and Open Society. En Lathrop, D. y Ruma, L. (Eds.), Open Government. Collaboration, Transparency, and Participation in Practice. Sebastopol: O'Reilly.

Fung, A., Mary Graham, M. y Weil, D. (2007). Full Disclosure: The Perils and Promise of Transparency. Cambridge: Cambridge University Press.

Fox, J. (2008). Transparencia y Rendición de Cuentas. En Ackerman, J. (Coord.), Más Allá del Acceso a la Información. Transparencia, Rendición de Cuentas y Estado de Derecho. México: Siglo XXI Editores. 
Geyer, R. y Rihani, S. (2010). Complexity and Public Policy. A New Approach to $21^{\text {st }}$ Century Politics, Policy and Society. Londres: Routledge.

Grimmelikhuijsen, S. (2012). Linking Transparency, Knowledge and Citizen Trust in Government: An Experiment. International Review of Administrative Sciences, 78 (1), 50-73.

Guardián Orta, C. (2010). ¿Transparencia? En Calderón, C. y Lorenzo, S. (Coords.), Open Government. Gobierno Abierto. Jaén: Algón Editores.

Harrison, T., Guerrero, S., Burk, G. y Cook, M. (2011). Open Government and E-Government: Democratic Challenges from a Public Value Perspective. Ponencia presentada en The 12th Annual International Digital Government Research Conference, 12-15 de junio, College Park, Maryland, Estados Unidos.

Heald, D. (2012). Why is Transparency about Public Expenditure so Elusive? International Review of Administrative Sciences, 78 (1), 30-49.

Jordana, J. (2007). El Análisis de los Policy Networks: ¿Una Nueva Perspectiva sobre la Relación entre Políticas Públicas y el Estado?. En Proyecto de Modernización del Estado (Ed.), Lecturas sobre el Estado y las Políticas Públicas. Retomando el Debate de Ayer para Fortalecer el Actual. Buenos Aires: Jefatura de Gabinete de Ministros, República de Argentina.

Kim, S. (2005) The Role of Trust in the Modern Administrative State: An Integrative Model. Administration \& Society, 37 (5), 611-635.

Kooiman, J. (2004). Gobernar en Gobernanza. Revista Instituciones y Desarrollo, 16, 171-194.

Lathrop, D. y Ruma, L. (Eds.). (2010). Open Government. Collaboration, Transparency, and Participation in Practice. Sebastopol: O'Reilly.

Linares, J. (2010). Las 10 Claves para Entender la Colaboración en el Modelo de Open Goverment. En Calderón, C. y Lorenzo, S. (Coords.), Open Government. Gobierno Abierto. Jaén: Algón Editores.

López Ayllón, S., Merino, M. y Morales, L. (2011). Hacia una Politica de Rendición de Cuentas en México. México: CIDE-Red para la Rendición de Cuentas-ASF.

Mariñez Navarro, F. y Valenzuela Mendoza, R. (2013). Gobierno Abierto. ¿Más Innovación? ¿Más Gobierno? ¿Más Sociedad? ¿En Qué Consiste? 
México: Editorial Miguel Angel Porrúa-EGAP.

Majone, G. (2005). Evidencia, Argumentación y Persuasión en la Formulación de Políticas. México: Fondo de Cultura Económica-Colegio Nacional de Ciencias Políticas y Administración Pública, A.C.

Meijer, A., Curtin, D, y Hillebrandt, M. (2012). Open Government: Connecting Vision and Voice. International Review of Administrative Sciences, 78 (1), 10-29.

Meijer, A. (2012). Introduction to the Special Issue on Government Transparency. International Review of Administrative Sciences, 78 (1), 3-9,

Nan, T. (2012). Citizens' Attitudes Toward Open Government and Government 2.0. International Review of Administrative Sciences, 78 (2). 346-368.

Noveck, B. (2010). The Single Point of Failure. En Lathrop, D. y Ruma, L. (Eds.), Open Government. Collaboration, Transparency, and Participation in Practice. Sebastopol: O’Reilly.

O'Reilly, T. (2010). Government as a Platform. En Lathrop, D. y Ruma, L. (Eds.), Open Government. Collaboration, Transparency, and Participation in Practice. Sebastopol: O’Reilly.

Papenful, U. y Schaefer, C. (2010). Improving Public Accountability by Aligning Reporting to Organizational Changes in Public Service Provision - An Empirical Internet Study of all Austrian, German and Swiss Towns and States from an Agency-Theory Perspective. International Review of Administrative Sciences, 76 (3), 555-576,

Park, H. y Blenkinsopp, J. (2011). The Roles of Transparency and Trust in the Relationship between Corruption and Citizen Satisfaction. International Review of Administrative Sciences, 77 (2), 254-274

Piotrowski, Suzanne J (2007). Government Transparency in the Path of Administrative Reform. Nueva York: The State of University of New York Press.

Pollitt, C. (2011). Mainstreaming Technological Change in the Study of Public Management. Public Policy and Administration, 26 (4), 377397.

Ramírez Alujas, A. (2011). Gobierno Abierto y Modernización de la 
Gestión Pública: Tendencias Actuales y el (Inevitable) Camino que Viene. Reflexiones Seminales. Revista Enfoques, IX (15), 99-125.

Schuler, D. (2010). Online Deliberation and Civic Intelligence. En Lathrop, D. y Ruma, L. (Eds.), Open Government. Collaboration, Transparency, and Participation in Practice. Sebastopol: O'Reilly.

Simmons, R., Birchall, J. y Prout, A. (2012). User Involvement in Public Services: 'Choice about Voice'. Public Policy and Administration, 27 (1), 3-29.

Stone, D. (2012). Policy Paradox. Nueva York: W.W. Norton \& Company.

Van der Meer, T. (2010). In What We Trust? A Multi-Level Study into Trust in Parliament as an Evaluation of State Characteristics. International Review of Administrative Sciences, 76 (3), 517-536

Recibido: 26-02-2013

Aceptación de la versión final: 29-11-2013 